\title{
Epigastric Hernia
}

\author{
Carlos Enrique Suarez Acosta ${ }^{1}$ - Esperanza Romero Fernandez ${ }^{1}$. \\ Elpidio Calvo Manuel $^{1}$
}

Received: 31 March 2015 / Accepted: 11 May 2015 / Published online: 23 May 2015

(C) Association of Surgeons of India 2015

\begin{abstract}
Epigastric hernia is a common condition, mostly asymptomatic although sometimes their unusual clinical presentation still represents a diagnostic dilemma for clinician. The theory of extra tension in the epigastric region by the diaphragm is the most likely theory of epigastric hernia formation. A detailed history and clinical examination in our thin, elderly male patient who presented with abdominal pain and constipation of 5 days of evolution was crucial in establishing a diagnosis. Noninvasive radiologic modalities such as ultrasonographic studies in the case of our patient can reliably confirm the diagnosis of epigastric hernia.
\end{abstract}

Keywords Epigastric · Hernia · Ultrasonography · Carlos Enrique · Suárez Acosta

\section{Epigastric Hernia}

Epigastric hernia is a common condition, mostly asymptomatic although sometimes their unusual clinical presentation still represents a diagnostic dilemma for clinician. The theory of extra tension in the epigastric region by the diaphragm is the most likely theory of epigastric hernia formation. A detailed history and clinical examination in our thin, 92-year-old male patient who presented with epigastric mass as shown in Fig. 1, abdominal pain, constipation of 5 days of evolution and decreased bowel sounds was crucial in establishing a diagnosis. Noninvasive radiologic modalities such as ultrasonographic studies as shown in Fig. 2, in the case of our patient, can reliably confirm the diagnosis of epigastric hernia. The patient received surgical treatment with good clinical evolution.

Carlos Enrique Suarez Acosta carlesuac1@yahoo.es

1 University Hospital Clinico San Carlos, Complutense University from Madrid, C/Profesor Martin Lagos s/n, PC 28040 Madrid, Spain

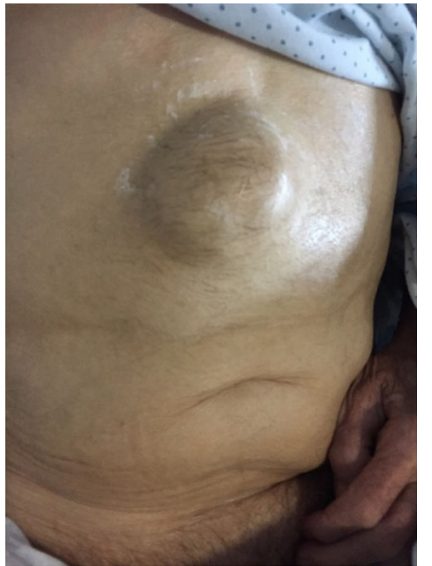

Fig. 1 Epigastric mass measuring $14 \times 10 \mathrm{~cm}$ in an elderly male patient with acute abdominal pain

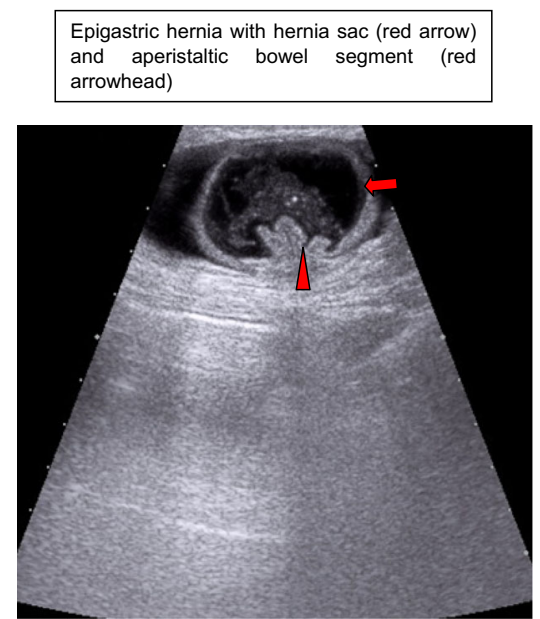

Fig. 2 Ultrasonographic study showing epigastric hernia with a hole measuring $2.3 \mathrm{~cm}$ on subxiphoid area and surrounded aperistaltic bowel segment suggestive of epigastric hernia incarcerated

Conflict of Interest The authors declare that they have no competing of interests. 